\title{
Neutrophil-mediated mechanisms in non-vascular Behçet's syndrome
}

\author{
Alessandra Bettiol ${ }^{1}$, MATTEO BECATTI ${ }^{2}$, Elena Slvestri ${ }^{1}$, Flavia Rita Argento ${ }^{2}$, \\ Eleonora Fini ${ }^{2}$, Amanda Mannucci², Silvia Galora ${ }^{2}$, Irene Mattioli², Maria Letizia Urban ${ }^{2}$, \\ Danilo Malandrino ${ }^{2}$, Adalgisa Palermo ${ }^{2}$, Niccolò Taddei ${ }^{1}$, Giacomo Emmi ${ }^{2}$, Domenico \\ Prisco $^{3}$, and Claudia Fiorillo ${ }^{1}$ \\ ${ }^{1}$ University of Firenze \\ ${ }^{2}$ University of Florence \\ ${ }^{3}$ University of Florence, Italy
}

August 3, 2021

\begin{abstract}
Objective: Behçet's syndrome (BS) is a systemic vasculitis with several clinical manifestations. Neutrophils hyperactivation mediates vascular BS involvement, via a massive production of reactive oxygen species (ROS) and the release of neutrophil extracellular traps (NETs). We investigated neutrophil-mediated mechanisms of damage in non-vascular BS manifestations and explored in vitro the effects of colchicine in counteracting these mechanisms. Methods: NETs and intracellular ROS production was assessed in blood samples from 80 BS patients (46 with active non-vascular BS, 34 with inactive disease) and 80 healthy controls. Moreover, isolated neutrophils were incubated for 1 hour with an oxidating agent (2,2'-azobis (2-amidinopropane) dihydrochloride; $250 \mathrm{nM}$ ), and the ability of pure colchicine pre-treatment $(100 \mathrm{ng} / \mathrm{ml})$ to counteract oxidation-induced damage was assessed. Results: Patients with active non-vascular BS had remarkably increased NET levels [21.2 (IQR 18.3-25.9) mU/ml] compared to patients with inactive disease $[16.8(13.3-20.2) \mathrm{mU} / \mathrm{ml}]$ and to controls $[7.1(5.1-8.7) \mathrm{mU} / \mathrm{ml}], \mathrm{p}<0.001]$. Also, intracellular ROS tended to be increased in active BS, although not significantly. In active non-vascular BS, NETs correlated with neutrophils ROS production $(\mathrm{p}<0.001)$ and were particularly increased in patients with active mucosal $(\mathrm{p}<0.001)$, articular $(\mathrm{p}=0.004)$, and gastrointestinal symptoms $(\mathrm{p}=0.006)$. On isolated neutrophils, colchicine significantly reduced oxidation-induced NET production and cell apoptosis, though not via an antioxidant activity. Conclusion: Neutrophil-mediated mechanisms might be directly involved in non-vascular BS, and NETs, more than ROS, might drive the pathogenesis of mucosal, articular and intestinal manifestations. Colchicine might be effective to counteract neutrophils-mediated damage in BS, although further studies are needed.
\end{abstract}

\section{Hosted file}

Manuscript_30.07.2021.docx available at https://authorea.com/users/428918/articles/532711neutrophil-mediated-mechanisms-in-non-vascular-beh\%C3\%A7et-s-syndrome 
a)

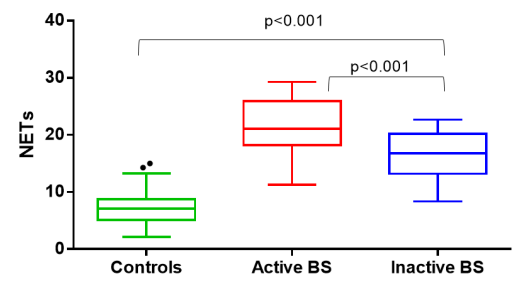

b)

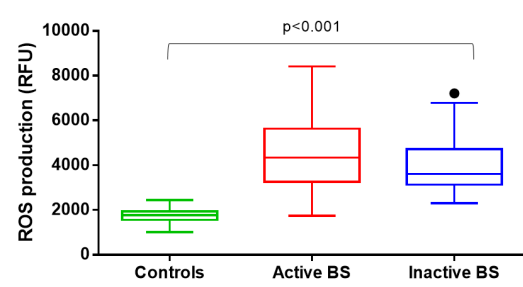

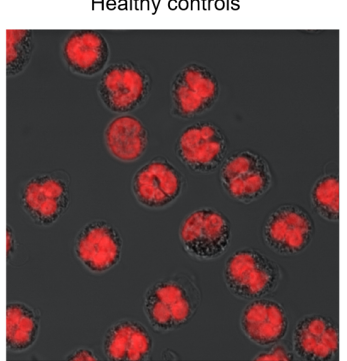

Active non-vascular BS

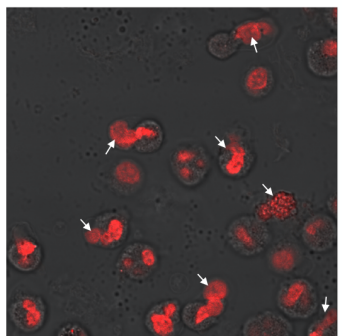

Inactive BS
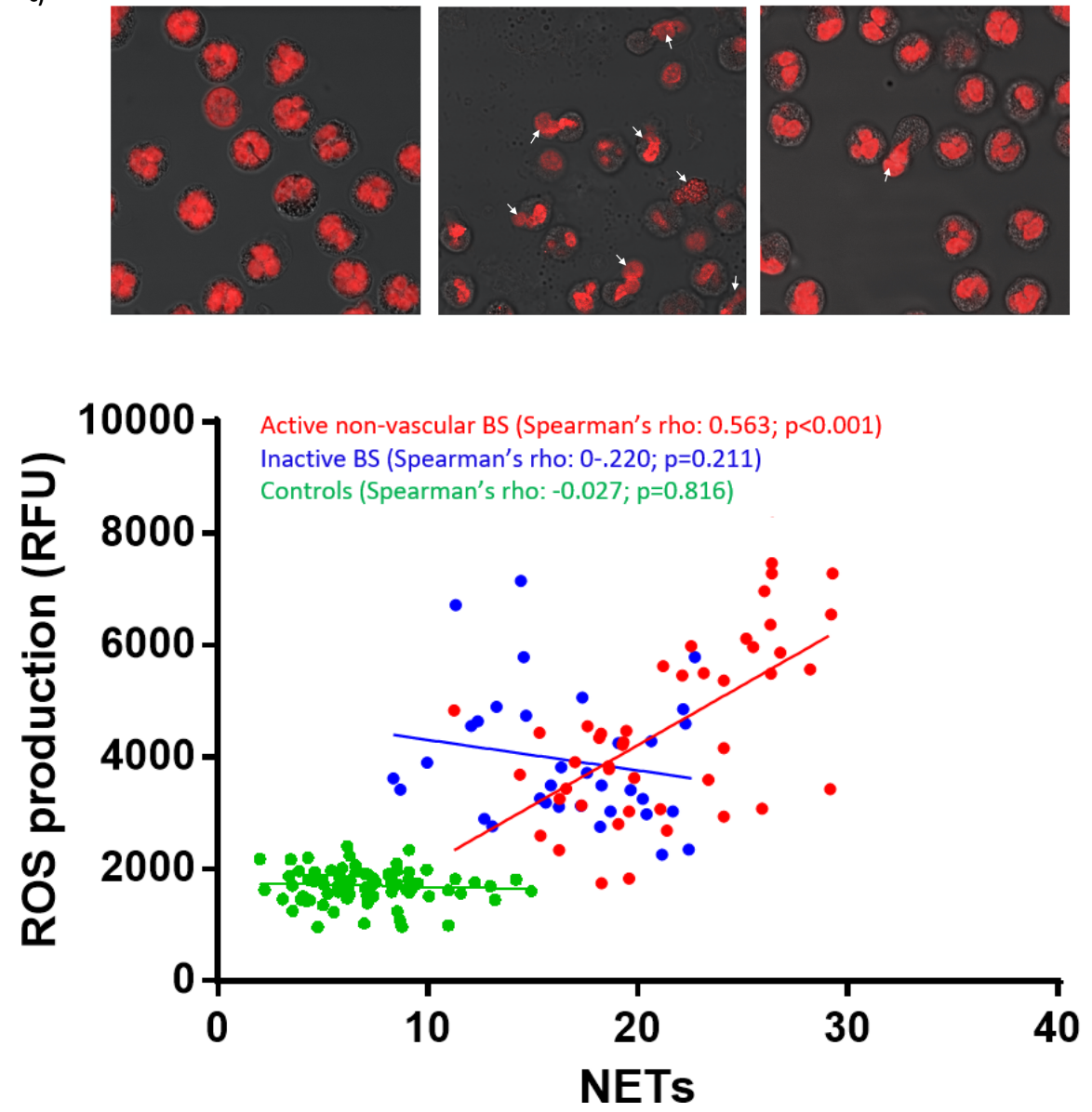

a)

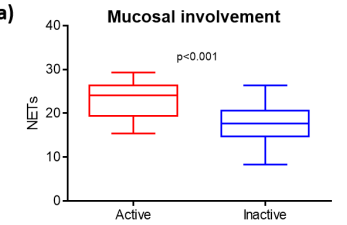

d)

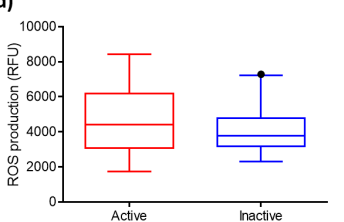

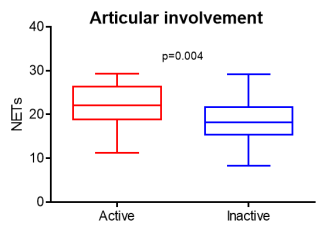

e)

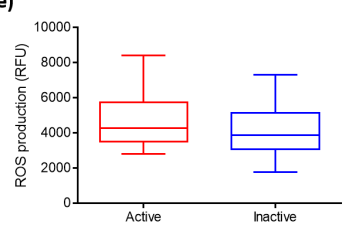

c)

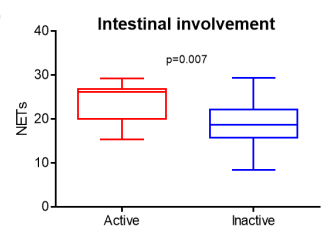

f)

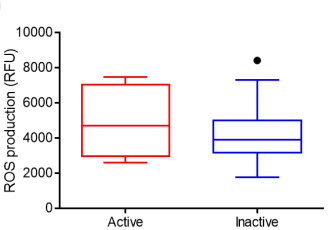


a)

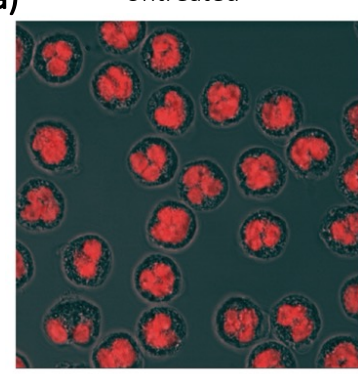

b)

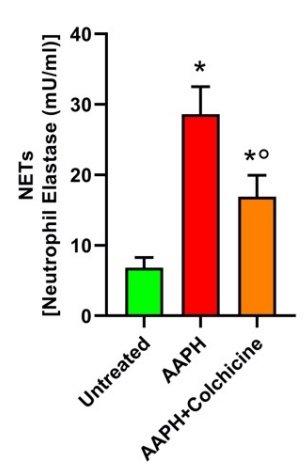

AAPH

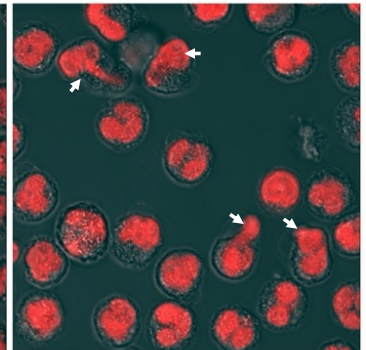

AAPH + Colchicine

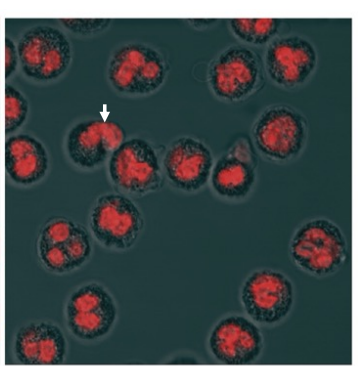

c)

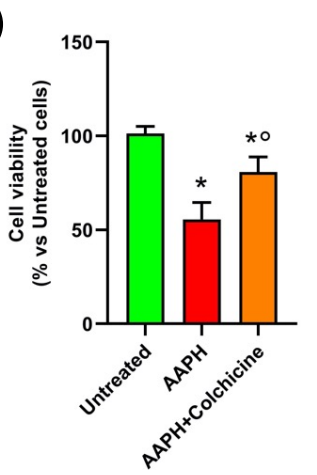

d)

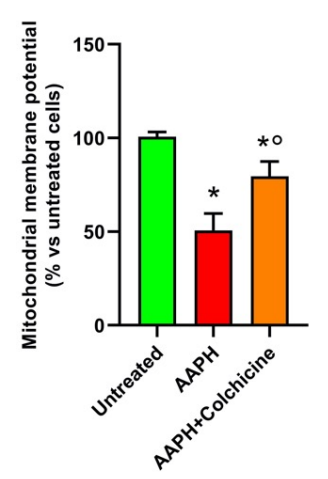

